Pacific Journal of Mathematics

BUNDLES OVER CONFIGURATION SPACES

FR DERICK RONALD COHEN, RALPH COHEN, 


\title{
BUNDLES OVER CONFIGURATION SPACES
}

\author{
F. R. Cohen, R. L. Cohen, N. J. Kuhn And J. L. Neisendorfer
}

Let $F\left(R^{n}, k\right)$ be the configuration space of ordered sets of $k$ distinct points in $R^{n}$. $F\left(R^{n}, k\right)$ is acted upon freely by the symmetric group on $k$ letters, $\Sigma_{k}$. In this paper we calculate the order of the vector bundles

$$
\xi_{n, k}: F\left(R^{n}, k\right) \times_{\Sigma_{k}} R^{k} \rightarrow F\left(R^{n}, k\right) / \Sigma_{k} .
$$
discussed.

Applications to the study of iterated loop spaces of spheres are also

1. The study of the stable homotopy type of the spaces $\Omega^{n} S^{n+r}$ has received much attention in recent years $[2,8,13]$. The starting point for this study was Snaith's stable descomposition [18]:

$$
\Omega^{n} S^{n+r} \simeq s \underset{k \geq 0}{\bigvee} F\left(\mathbf{R}^{n}, k\right)^{+} \wedge_{\Sigma_{k}} S^{r^{(k)}},
$$

where $F\left(\mathbf{R}^{n}, k\right)^{+}$is the configuration space of $k$ ordered distinct points in $\mathbf{R}^{n}$ together with a disjoint basepoint, $S^{r^{(k)}}$ is the $k$-fold smash product of $S^{r}$ with itself, $\Sigma_{k}$ is the symmetric group of $k$ letters, and where " $\simeq_{s}$ " denotes stable homotopy equivalence.

The space $F\left(\mathbf{R}^{n}, k\right)^{+} \wedge_{\Sigma_{k}} S^{r^{(k)}}$ is clearly the Thom complex of the $r$-fold Whitney sum of the vector bundle

$$
\xi_{n, k}: F\left(\mathbf{R}^{n}, k\right) \times_{\Sigma_{k}} \mathbf{R}^{k} \rightarrow F\left(\mathbf{R}^{n}, k\right) / \Sigma_{k} .
$$

If $M\left(\xi_{n, k}\right)$ is the associated Thom spectrum, then Snaith's theorem gives an equivalence of spectra

$$
\Sigma^{\infty} \Omega^{n} S^{n+r} \simeq \underset{k \geq 0}{\bigvee} \Sigma^{r k} M\left(r \xi_{n, k}\right)
$$

where $\Sigma^{\infty}$ is the stabilization functor which assigns to a space its associated suspension spectrum.

If $\phi_{n, k}$ is the stable order of $\xi_{n, k}$ (i.e., $\phi_{n, k}$ is the smallest integer such that $\phi_{n, k} \xi_{n, k}$ is stably trivial) then we have the obvious periodicity

$$
M\left(\left(r+\phi_{n, k}\right) \xi_{n, k}\right) \simeq \sum^{k \phi_{n, k}} M\left(r \xi_{n, k}\right) .
$$

This, together with Snaith's theorem gives clear interrelationships amongst the stable homotopy types of the spaces $\Omega^{n} S^{n+r}$ as $r$ varies.

The case $n=2$ is well understood by the work of F. Cohen, M. Mahowald, and R. J. Milgram [5], who proved that $\phi_{2, k}=2$ for all $k$. The resulting periodicity in the homotopy type of the associated Thom 
spectra was used by M. Mahowald [13] and R. Cohen [8] to construct new infinite families in the stable homotopy ring $\pi_{*}^{s}$.

It is the purpose of this paper to compute the orders $\phi_{n, k}$ for general $n$ and $k$. Our main result can be stated as follows. Let

$$
a_{n, k}=2^{\rho(n-1)} \prod_{3 \leq p \leq k} p^{[(n-1) / 2]}
$$

where $p$ denotes an odd prime, and where $\rho(m)$ is Adam's vector field number: $\rho(m)=$ the number of positive integers $\leq m$ that are congruent to $0,1,2$, or $4 \bmod 8$.

THEOREM 1.1. If $n \neq 0 \bmod 4$, then $\phi_{n, k}=a_{n, k}$. Furthermore, if $n \equiv 0 \bmod 4$, then $a_{n, k} \mid \phi_{n, k}$ and $\phi_{n, k} \mid 2 a_{n, k}$.

REMARKS. 1. The bundle $\xi_{n, 2}$ is easily seen to be stably isomorphic to the canonical line bundle over $\mathbf{R} P^{n-1}$, so the fact that $\phi_{n, 2}=2^{\rho(n-1)}$ is the classical result of Adams [1].

2. Using the Atiyah-Hirzebruch spectral sequence converging to the KO-theory of $F\left(\mathbf{R}^{n}, p\right) / \Sigma_{p}, \mathrm{~S}$. W. Yang computed the order of $\xi_{n, p}$, and proved that $a_{n, k} \mid \phi_{n, k}[\mathbf{2 0}]$.

3. The conjecture that $\phi_{n, k}=a_{n, k}$ was made by Yang, Mahowald, and F. Cohen.

The essential idea in the proof of 1.1 is to notice that the classifying map

$$
f_{n, k}: F\left(\mathbf{R}^{n}, k\right) / \Sigma_{k} \rightarrow B O
$$

of $\xi_{n, k}$ factors as a composition of maps, one of which is the natural inclusion

$$
i_{n}: \Omega_{0}^{n} S^{n} \hookrightarrow Q_{0} S^{0}
$$

where $Q X=\lim _{m \rightarrow \infty} \Omega^{m} \Sigma^{m} X$, and where $\Omega_{k}^{n} S^{n}$ denotes the component of $\Omega^{n} S^{n}$ containing maps of degree $k$. We then study the order of $i_{n}$ localized at a prime $p$, using the results of F. Cohen, J. Moore, and J. Neisendorfer $[6,7,15]$ and of Toda [19].

AcKnowledgements. The authors are grateful to M. Mahowald, J. P. May, and L. Traylor for helpful conversations concerning the material in this paper. 
2. Proof of Theorem 1.1. Our first object is to identify the classifying maps of the bundles $\xi_{n, k}$. This is done easily by recalling first that $F\left(\mathbf{R}^{\infty}, k\right)=\lim _{n \rightarrow \infty} F\left(\mathbf{R}^{n}, k\right)$ is a contractible space, acted upon freely by $\Sigma_{k}$, and therefore $F\left(\mathbf{R}^{\infty}, k\right) / \Sigma_{k}=B \Sigma_{k}$. For a proof of this, see for instance [14].

Thus the bundle

$$
\xi_{\infty, k}: F\left(\mathbf{R}^{\infty}, k\right) \times_{\Sigma_{k}} \mathbf{R}^{k} \rightarrow F\left(\mathbf{R}^{\infty}, k\right) / \Sigma_{k}=B \Sigma_{k}
$$

is classified by the map

$$
f_{k}: B \Sigma_{k} \rightarrow B O(k)
$$

induced by the regular representation of $\Sigma_{k}$ in $O(k)$. Moreover, since the bundle $\xi_{n, k}$ is the pull-back of $\xi_{\infty, k}$ under the inclusion $F\left(\mathbf{R}^{n}, k\right) / \Sigma_{k} \subset$ $F\left(\mathbf{R}^{\infty}, k\right) / \Sigma_{k}, \xi_{n, k}$ is classified by the map

$$
f_{n, k}: F\left(\mathbf{R}^{n}, k\right) / \Sigma_{k} \subset F\left(\mathbf{R}^{\infty}, k\right) / \Sigma_{k}=B \Sigma_{k} \underset{f_{k}}{\rightarrow} B O(k) .
$$

The stable order $\phi_{n, k}$ of $\xi_{n, k}$ is the order of the class determined by $f_{n, k}$ in the abelian group $\left[F\left(\mathbf{R}^{n}, k\right) / \Sigma_{k}, B O\right]$. In order to determine $\phi_{n, k}$ we first recall some of May's iterated loop space machinery [14].

Recall first the "approximations"

$$
\alpha_{n}: C_{n} X \rightarrow \Omega^{n} \sum^{n} X
$$

of [14]. $C_{n} X$ is a filtered space which approximates $\Omega^{n} \sum^{n} X$ in the sense that $\alpha_{n}$ is a weak homotopy equivalence if $X$ is connected. For $X=S^{0}$,

$$
C_{n}\left(S^{0}\right) \simeq \amalg_{k} F\left(\mathbf{R}^{n}, k\right) / \Sigma_{k}
$$

and the map $\alpha_{n}: \amalg_{k} F\left(\mathbf{R}^{n}, k\right) / \Sigma_{k} \rightarrow \Omega^{n} S^{n}$ takes $F\left(\mathbf{R}^{n}, k\right) / \Sigma_{k}$ to $\Omega_{k}^{n} S^{n}$.

Now consider the map

$$
\beta: \underset{k}{\amalg} B O(k) \rightarrow B O \times \mathbf{Z}
$$

which includes $B O(k)$ into $B O \times\{k\}$ in the obvious manner. Let $\eta: Q S^{0} \rightarrow B O \times \mathbf{Z}$ be the infinite loop map induced by the map $S^{0} \rightarrow$ $B O \times \mathbf{Z}$ which sends 0 to the basepoint in $B O \times\{0\}$ and 1 to the basepoint in $B O \times\{1\}$. We then have

LEMMA 2.1. The following diagram homotopy commutes for all positive integers $n$ and $k$. 


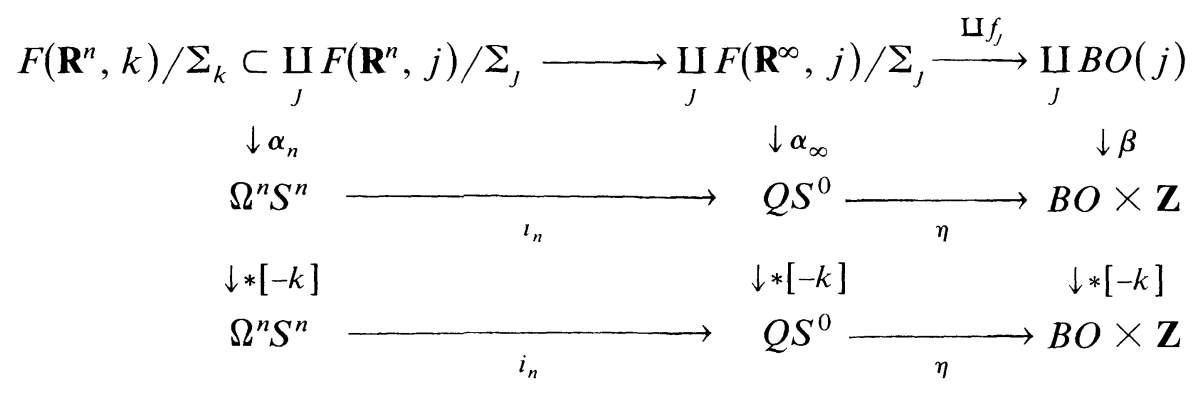

where $*[-k]$ translates components by $-k$.

Proof. This follows directly from May's iterated loop space machinery, and an explicit proof is found in [4].

Note that the classifying map $f_{n, k}: F\left(\mathbf{R}^{n}, k\right) / \Sigma_{k} \rightarrow B O=B O \times\{0\}$ $\subset B O \times \mathbf{Z}$ of $\xi_{n, k}$ is the composition obtained by going along the top and then down the right-hand side of the diagram in Lemma 2.1. Now since $\eta$ is a map of infinite loop spaces, and therefore like $i_{n}$ is an $H$-map, Lemma 2.1 implies that the power of $p$ in the prime factorization of $\phi_{n, k}$ is bounded by the order of the localization at $p$ of $i_{n} \in\left[\Omega_{0}^{n} S^{n}, Q_{0} S^{0}\right]$.

Proposition 2.2. For a prime $p$, let $i_{n, p}: \Omega_{0}^{n} S_{(p)}^{n} \rightarrow Q_{0} S_{(p)}^{0}$ be the localization of $i_{n}$. Then in $\left[\Omega_{0}^{n} S_{(p)}^{n}, Q_{0} S_{(p)}^{0}\right]$ the order of $i_{n, p}$ divides $p^{q}$, where

$$
q= \begin{cases}{\left[\frac{n-1}{2}\right]} & \text { if } p \text { is odd } \\ \rho(n-1) & \text { if } p=2 \text { and } n \neq 0 \bmod 4 \\ \rho(n-1)+1 & \text { if } p=2 \text { and } n \equiv 0 \bmod 4\end{cases}
$$

Notice that Theorem 1.1 is a corollary of Proposition 2.2 in view of Yang's results [20] (see the second remark following the statement of Theorem 1.1), and the fact that if $k<p, F\left(\mathbf{R}^{\infty}, k\right) / \Sigma_{k}=B \Sigma_{k}$ is homology $p$-equivalent to a point.

Proof of 2.2. We prove Proposition 2.2 in several cases.

Case 1. $p$ odd and $n$ odd (say $n=2 m+1$ ).

Recent results of Selick [17], Cohen, Moore and Neisendorfer [6, 7], and Neisendorfer [15] imply that the identity element

$$
1 \in\left[\Omega_{0}^{2 m+1} S_{(p)}^{2 m+1}, \Omega_{0}^{2 m+1} S_{(p)}^{2 m+1}\right]
$$

has order $p^{m}$. Since $i_{n}$ is an $H$-map, the result follows in this case. 
Case 2. $p=2, n$ odd.

To verify this case we shall use the Kahn-Priddy theorem [10]:

THEOREM 2.3. There exist maps $s: Q \mathbf{R} P^{\infty} \rightarrow Q_{0} S^{0}$ and $j: Q_{0} S^{0} \rightarrow$ $Q \mathbf{R} P^{\infty}$ such that when localized at the prime $2, s \circ j$ is a homotopy equivalence.

In [16], Segal gave a proof of this theorem in which he showed that when restricted to $\Omega_{0}^{n} S^{n} \subset Q_{0} S^{0}, j$ factors through a map $j_{n}: \Omega_{0}^{n} S^{n} \rightarrow$ $Q \mathbf{R} P^{n-1}$. In [3], Caruso, Cohen, May, and Taylor also gave a proof of the Kahn-Priddy theorem, obtaining Segal's factorization, and in which explicit formulae for the maps $j_{n}, j$, and $s$ are given.

In any case, using the proof and the formulae in [3] of this theorem, N. Kuhn verified that the maps $j_{n}$ and $j$ are one-fold loop maps [12]. The fact that $j$ is an $H$-map actually follows immediately from Kahn's work in [11]. Using these results, we shall consider the following homotopy commutative diagram of spaces localized at 2.

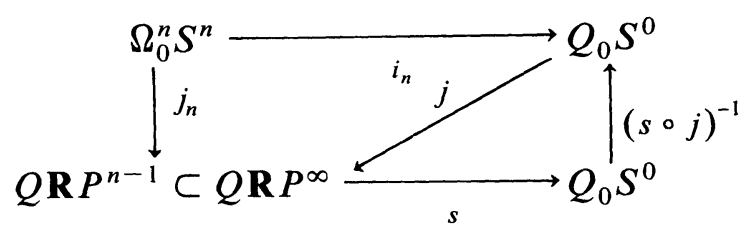

where $(s \circ j)^{-1}$ is a homotopy inverse to $s \circ j$. Since $s$ is an infinite loop map, and $j$ deloops once, $s \circ j$ and therefore $(s \circ j)^{-1}$ are maps of loop spaces. Thus the order of $i_{n}$ (localized at 2) divides the order of the identity of $Q \mathbf{R} P^{n-1}$, which Toda showed to be $2^{\rho(n-1)}$ when $n$ is odd [19]. This proves the proposition in this case.

Case 3. $n=2 m$.

Consider the following fibration of James [9].

$$
S^{2 m-1} \stackrel{e}{\rightarrow} \Omega S^{2 m} \stackrel{h}{\rightarrow} \Omega S^{4 m-1}
$$

This fibration yields the classical EHP sequence in homotopy groups. Apply $\Omega^{2 m-1}$ to this fibration and consider the following diagram. 


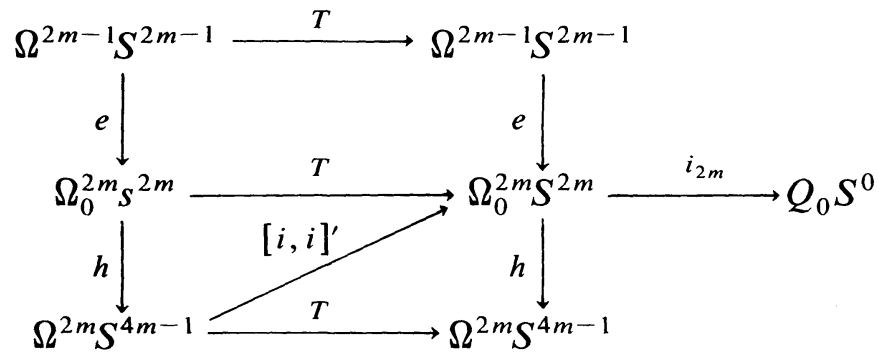

where $T$ is twice the identity map, and $[i, i]^{\prime}=\Omega^{2 m}[i, i]$, where $[i, i]$ : $S^{4 m-1} \rightarrow S^{2 m}$ is the Whitehead product of the identity with itself.

LEMMA 2.4. In the above diagram we have

(a) both squares commute,

(b) the lower triangle commutes, and

(c) $i_{2 m} \circ[i, i]^{\prime}$ is null homotopic.

Proof. The commutativity of the two squares is obvious, and the commutativity of the lower triangle follows from the standard fact that the Hopf invariant of $[i, i]$ is 2. Similarly, the fact that $i_{2 m} \circ[i, i]^{\prime}=0$ follows from the standard fact that the Whitehead product $[i, i]$ stabilizes to zero.

COROLlaRY 2.5. There exists a map g: $\Omega_{0}^{2 m} S^{2 m} \rightarrow \Omega_{0}^{2 m-1} S^{2 m-1}$ so that $T \simeq[i, i]^{\prime} \circ h+e \circ g$.

Proof. By the lemma, $h \circ\left(T-[i, i]^{\prime} \circ h\right)$ is null homotopic, and therefore $T-[i, i]^{\prime} \circ h$ lifts to a map $g: \Omega_{0}^{2 m} S^{2 m} \rightarrow S^{2 m-1}$ satisfying the required property.

We are now ready to prove the proposition in this final case. Localizing at 2 , we have that

$$
\begin{aligned}
2^{\rho(2 m-2)+1} i_{2 m} & =2^{\rho(2 m-2)}\left(i_{2 m} \circ T\right) \\
& =2^{\rho(2 m-2)}\left(i_{2 m} \circ[i, i]^{\prime} \circ h+i_{2 m} \circ e \circ g\right)
\end{aligned}
$$

by 2.5 , and which equals $2^{\rho(2 m-2)}\left(i_{2 m-1} \circ g\right)$ by 2.4 part $\mathrm{c}$ and the fact that $i_{2 m-1}=i_{2 m} \circ e$. But $2^{\rho(2 m-2)} i_{2 m-1}$ is null homotopic by the result in case 2 . We may therefore conclude that

$$
2^{\rho(2 m-2)+1} i_{2 m}=0 .
$$

Similarly, localized at $p$ odd and using the result of case 1 , we obtain that $2 p^{[(n-1) / 2]} i_{2 m}$ is null homotopic, and therefore so is $p^{[(n-1) / 2]} i_{2 m}$. 
Thus we have proved the proposition when $p$ is odd, and summarizing the results in $p=2$, we have:

$$
\begin{array}{ll}
2^{\rho(n-1)} i_{n}=0 & \text { if } n \text { is odd, } \\
\text { and } \quad 2^{\rho(n-2)+1} i_{n}=0 & \text { if } n \text { is even, } \\
2_{n}=0 & \text { if } n \text { is even. }
\end{array}
$$

The last equation follows from the first since $i_{2 m}$ factors through $i_{2 m+1}$.

Notice that if $n \equiv 2 \bmod 8, \rho(n-1)=\rho(n-2)+1$ and therefore $2^{\rho(n-1)} i_{n}=0$. If $n \equiv 6 \bmod 8, \rho(n-1)=\rho(n)$ so $2^{\rho(n-1)} i_{n}=0$. Thus if $n \neq 0 \bmod 4,2^{\rho(n-1)} i_{n}$ is null homotopic. If $n \equiv 0 \bmod 4, \rho(n-1)=$ $\rho(n-2)$ so $2^{\rho(n-1)+1} i_{n}=0$.

This completes the proof of Proposition 2.2, and therefore of Theorem 1.1.

\section{REFERENCES}

1. J. F. Adams, Vector fields on spheres, Annals of Math., (2) 75 (1962), 603-632.

2. E. H. Brown, Jr. and F. P. Peterson, On the stable decomposition of $\Omega^{2} S^{r+2}$, Trans. Amer. Math. Soc., 243 (1978), 287-298.

3. J. Caruso, F. Cohen, J. P. May, and L. Taylor, James maps, Segal maps, and the Kahn-Priddy theorem, to appear.

4. F. R. Cohen, Braid orientations and bundles with flat connections, Invent. Math., 46 (1978), 99-110.

5. F. R. Cohen, M. Mahowald, and R. J. Milgram, The stable decomposition of the double loop space of a sphere, Proc. Symp. Pure Math., Vol. 32 part 2 (1978), 225-228.

6. F. R. Cohen, J. Moore, and J. Neisendorfer, Torsion in homotopy groups, Annals of Math. 109, (1979), 121-168.

7. The double suspension and exponents of the homotopy groups of spheres, to appear in Annals of Math.

8. R. L. Cohen, Odd primary infinite families in stable homotopy theory, Memoirs of the A.M.S., Vol. 30 No. 242 (1981).

9. I. M. James, On the suspension sequence, Annals of Math., (2) 65 (1957), 74-107.

10. D. Kahn and S. Priddy, The transfer and stable homotopy theory, Math. Proc. Camb. Phil. Soc., 83 (1978), 103-12.

11. D. S. Kahn, Homology of the Barratt-Eccles decomposition maps, Proc. Conf. on Homotopy Theory, Northwestern Univ. Notas de Mat. y. Simposia No. 1, Soc. Mat. Mexicana (1975), 60-77.

12. N. Kuhn, Ph.D. Dissertation, University of Chicago, 1980.

13. M. Mahowald, $A$ new infinite family in ${ }_{2} \pi_{*}^{s}$, Topology, 16 (1977), 249-254.

14. J. P. May, The Geometry of Iterated Loop Spaces, Springer Lecture Notes in Math., 271 (1972).

15. J. Neisendorfer, 3-primary exponents, to appear.

16. G. Segal, Operations in stable homotopy theory, New Developments in Topology, J. London Math. Soc. Lecture Notes, Vol. 11 (1974), 105-110.

17. P. Selick, Odd primary torsion in $\pi_{k}\left(S^{3}\right)$, Topology, 17 (1978), 407-412.

18. V. P. Snaith, A stable decomposition for $\Omega^{n} S^{n} X$, J. London Math. Soc., 7 (1974), $577-583$. 
54 F. R. COHEN, R. L. COHEN, N. J. KUHN AND J. L. NEISENDORFER

19. H. Toda, Order of the identity class of a suspension space, Annals of Math., (2) 78 (1963), 300-325.

20. S. W. Yang, Ph.D. Dissertation, Brandeis University, 1978.

Received July 7, 1980. The authors were partially supported by the National Science Foundation. The first author was partially supported by the Alfred Sloan Foundation.

UNIVERSITY OF KENTUCKY

LEXINGTON, KY 40506

STANFORD UNIVERSITY

STANFORD, CA 94305

UNIVERSITY OF WASHINGTON

Seattle, WA 98195

AND

OHio STATE University

Columbus, OH 43210 


\section{PACIFIC JOURNAL OF MATHEMATICS \\ EDITORS}

DONALD BABBITT (Managing Editor)

University of California

Los Angeles, CA 90024

\section{Hugo Rossi}

University of Utah

Salt Lake City, UT 84112

C. C. Moore and Arthur Ogus

University of California

Berkeley, CA 94720
J. DugundiI

Department of Mathematics

University of Southern California

Los Angeles, CA 90089-1113

R. FINN and H. SAMELSON

Stanford University

Stanford, CA 94305

ASSOCIATE EDITORS
R. ARens
E. F. BECKENBACH
B. H. NeumanN
F. WOLF
K. YoshidA (1906-1982)

\section{SUPPORTING INSTITUTIONS}

UNIVERSITY OF ARIZONA

UNIVERSITY OF BRITISH COLUMBIA

CALIFORNIA INSTITUTE OF TECHNOLOGY

UNIVERSITY OF CALIFORNIA

MONTANA STATE UNIVERSITY

UNIVERSITY OF NEVADA, RENO

NEW MEXICO STATE UNIVERSITY

OREGON STATE UNIVERSITY
UNIVERSITY OF OREGON

UNIVERSITY OF SOUTHERN CALIFORNIA

STANFORD UNIVERSITY

UNIVERSITY OF HAWAII

UNIVERSITY OF TOKYO

UNIVERSITY OF UTAH

WASHINGTON STATE UNIVERSITY

UNIVERSITY OF WASHINGTON 


\section{Pacific Journal of Mathematics}

Vol. 104, No. 1

May, 1983

Nestor Edgardo Aguilera and Eleonor Ofelia Harboure de Aguilera, On

the search for weighted norm inequalities for the Fourier transform $\ldots \ldots .1$

Jin Akiyama, Frank Harary and Phillip Arthur Ostrand, A graph and its complement with specified properties. VI. Chromatic and achromatic numbers ......................................... 15

Bing Ren Li, The perturbation theory for linear operators of discrete type . . . 29

Peter Botta, Stephen J. Pierce and William E. Watkins, Linear

transformations that preserve the nilpotent matrices .............. 39

Frederick Ronald Cohen, Ralph Cohen, Nicholas J. Kuhn and Joseph

Alvin Neisendorfer, Bundles over configuration spaces .......... 47

Luther Bush Fuller, Trees and proto-metrizable spaces . . . . . . . . . . 55

Giovanni P. Galdi and Salvatore Rionero, On the best conditions on the

gradient of pressure for uniqueness of viscous flows in the whole space . . 77

John R. Graef, Limit circle type results for sublinear equations $\ldots \ldots \ldots \ldots 85$

Andrzej Granas, Ronald Bernard Guenther and John Walter Lee,

Topological transversality. II. Applications to the Neumann problem for

$y^{\prime \prime}=f\left(t, y, y^{\prime}\right) \ldots \ldots \ldots \ldots \ldots \ldots \ldots \ldots \ldots \ldots \ldots \ldots \ldots \ldots \ldots . \ldots 5$

Richard Howard Hudson and Kenneth S. Williams, Extensions of

theorems of Cunningham-Aigner and Hasse-Evans . . . . . . . . . . . 111

John Francis Kurtzke, Jr., Centralizers of irregular elements in reductive algebraic groups

James F. Lawrence, Lopsided sets and orthant-intersection by convex

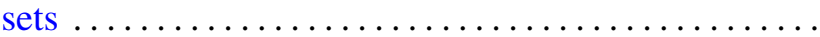

Åsvald Lima, G. H. Olsen and U. Uttersrud, Intersections of $M$-ideals and

$G$-spaces

Wallace Smith Martindale, III and C. Robert Miers, On the iterates of derivations of prime rings

Thomas H. Pate, Jr, A characterization of a Neuberger type iteration procedure that leads to solutions of classical boundary value problems

Carl L. Prather and Ken Shaw, Zeros of successive iterates of multiplier-sequence operators

Billy E. Rhoades, The fine spectra for weighted mean operators

Rudolf J. Taschner, A general version of van der Corput's difference theorem

Johannes A. Van Casteren, Operators similar to unitary or selfadjoint ones 